\title{
Evaluación del estudio pT3a de la actual clasificación TNM del cáncer renal
}

\author{
L. Gómez Pérez, A. Budía Alba, J.L. Pontones Moreno, F.J. Delgado Oliva, J.L. Ruíz Cerdá, \\ F. Jiménez Cruz
}

\author{
Servicio de Urología. Hospital Universitario La Fe. Valencia.
}

Actas Urol Esp 2006; 30 (3): 287-294

\section{RESUMEN}

EVALUACIÓN DEL ESTADIO pT3a DE LA ACTUAL CLASIFICACIÓN TNM DEL CÁNCER RENAL

Objetivo: Considerando los tumores renales en estadio pT3a por infiltración grasa, hemos evaluado dicho estadio en función del tamaño tumoral en nuestra serie.

Material y método: Hemos realizado un estudio retrospectivo de 300 pacientes con diagnóstico de adenocarcinoma renal (CCR) entre los años 1992 a 2001, con un seguimiento medio de 60 meses. Incluimos en nuestro estudio a 92 pacientes $(91,08 \%)$ de todos los tumores pT3a por infiltración grasa, independientemente de la afectación ganglionar (Ncualquiera) y con ausencia de metástasis. Se obtuvieron curvas ROC en función del tamaño tumoral y el estado (vivo/muerto), seleccionando el mejor valor de corte (mejor sensibilidad y especificidad). Dicho punto de corte correspondió al valor $5,5 \mathrm{~cm}$, permitiendo dicotomizar y definir dos grupos con diferencias significativas respecto a la supervivencia actuarial. Tras diferenciar estos dos grupos, comparamos los tumores menores de 5,5 cm y estadio pT3aNxM0, con el resto de subgrupos de la clasificación TNM.

Resultados: No encontramos diferencias significativas al comparar la supervivencia actuarial de los tumores pT3a seleccionados y tamaño inferior a $5,5 \mathrm{~cm}$ con el resto de tumores en estadios pT1 y pT2. Tras reclasificar los tumores pT3a menores de 5,5 cm como pT1, se realizó un análisis multivariante mediante regresión logística para evaluar los factores pronósticos respecto a la progresión tumoral, previo y posterior a la reclasificación de los tumores, conservando el TNM su valor pronóstico independiente tras la reclasificación.

Conclusiones: Los tumores de células renales pT3a por infiltración grasa sin metástasis de tamaño inferior a 5,5cm, se comportan como tumores organoconfinados respecto a la supervivencia y su reclasificación no modifica el valor pronóstico independiente del TNM como predictor de progresión tumoral.

Palabras clave: Cáncer renal.

\section{ABSTRACT}

EVALUATION OF pT3a STAGE IN THE ACTUAL CLASSIFICATION FOR RENAL CANCER

Objective: We assessed the prognostic value of a stage pT3a diagnosis based on perirrenal fat infiltration.

Material and methods: A series of 300 patients diagnosed of renal cell carcinoma (CCR) between 1992 and 2001 were retrospectively analyzed. Focusing on pT3a tumors as defined by perirrenal fat infiltration, a group of 92 patients $(91,08 \%)$ regardless lymph node involvement (Nall) were included. Patients with distant metastases were excluded. In patients with pT3a Nall M0 tumors, tumour size was a significant parameter predicting survival. The most significant cut-off value for tumor size based on ROC curve was $5,5 \mathrm{~cm}$. Therefore two groups were defined (up to $5,5 \mathrm{~cm}$ or greater than $5,5 \mathrm{~cm}$ ) and actuarial survival were compared between both groups.

Results: No significant differences were found comparing actuarial survival of selected pT3a and tumour size less than $5,5 \mathrm{~cm}$ with pT1 and pT2 tumors. After classifying selected pT3a less than $5,5 \mathrm{~cm}$ as pT1, multivariate analysis showed no differences regarding to prognostic variables before and after classification. Subsequently multivariate analysis showed that modified $\mathrm{T}$ stage was an independent significant predictor of cancer specific actuarial survival.

Conclusions: Perirrenal fat infiltration in renal cell carcinoma should not be used to assign $\mathrm{T}$ category. In our series grading tumors pT3a lesser than $5,5 \mathrm{~cm}$ as pT1/pT2 TNM stage does not affect their prognostic value.

Keywords: Renal cancer. 
$\mathrm{E}$ 1 objetivo inicial de la clasificación TNM para neoplasias malignas es la de describir la extensión anatómica de la enfermedad, evaluar el pronóstico, facilitar la comparación de resultados y ayudar en la decisión terapéutica ${ }^{1-3}$.

El estadiaje TNM para el cáncer de células renales ha sufrido modificaciones desde su primera edición. En la actual clasificación TNM 2002 , el estadio $T_{1}$ se reclasificó en dos subgrupos en función del tamaño, Tla (menor o igual a $4 \mathrm{~cm}) \mathrm{y} \mathrm{T}_{1 \mathrm{~b}}(\mathrm{de} 4$ a $7 \mathrm{~cm}$ ), definiéndose un punto de corte a partir del cual no era aconsejable el tratamiento quirúrgico conservador $(4 \mathrm{~cm})^{3}$. Sin embargo el estadio $\mathrm{T}_{3}$ no ha sido modificado. Este estadio incluye tumores que infiltran el tejido adiposo perirrenal y aquellos que invaden la glándula adrenal adyacente por crecimiento tumoral. En estudios recientes se ha apuntado que los tumores $\mathrm{pT}_{3 \mathrm{a}}$ por infiltración directa de la glándula adrenal ipsilateral y/o grasa perihiliar, tienen peor supervivencia que el resto de tumores $\mathrm{pT}_{3 \mathrm{a}}$ por infiltración grasa y que ésta es comparable a los pacientes con tumores $\mathrm{pT}_{4}$, por lo que proponen incluir estos tumores en un nuevo estadio $\mathrm{pT}_{4}$ reclasificado, dejando el estadio $\mathrm{pT}_{3 \mathrm{a}}$ para los tumores renales con infiltración grasa ${ }^{4}$.

En este estudio hemos evaluado los tumores renales $\mathrm{pT}_{3 \mathrm{a}} \mathrm{N}_{\mathrm{x}} \mathrm{M}_{0}$ por infiltración grasa según la clasificación TNM 2002 en función del tamaño, a fin de mejorar el valor pronóstico de la misma para este estadio.

\section{MATERIAL Y MÉTODO}

Hemos realizado un estudio retrospectivo de 300 pacientes con diagnóstico de adenocarcinoma renal (CR) intervenidos quirúrgicamente mediante nefrectomía radical y linfadenectomía entre los años 1992 a 2001.

Los tumores fueron reclasificados según la actual clasificación TNM 2002 como se muestra en la Fig. 1. El examen anatomopatológico clasificó en estadio $\mathrm{pT}_{3 \mathrm{a}}$ a 101 pacientes $(33,66 \%)$, con una mediana de edad de 68 años (rango 26 a 78). De éstos, $96(95,04 \%)$ eran $\mathrm{pT}_{3 \mathrm{a}}$ por afectación grasa y $5(4,95 \%)$ por invasión directa de glándula adrenal ipsilateral, (3 de ellos con metástasis al diagnóstico). De los 96 pacientes, 4 de ellos presentaban enfermedad a distancia y $23(23,95 \%)$ afectación ganglionar $\left(\mathrm{pT}_{3 \mathrm{a}} \mathrm{N}_{1} \mathrm{M}_{0}\right)$.

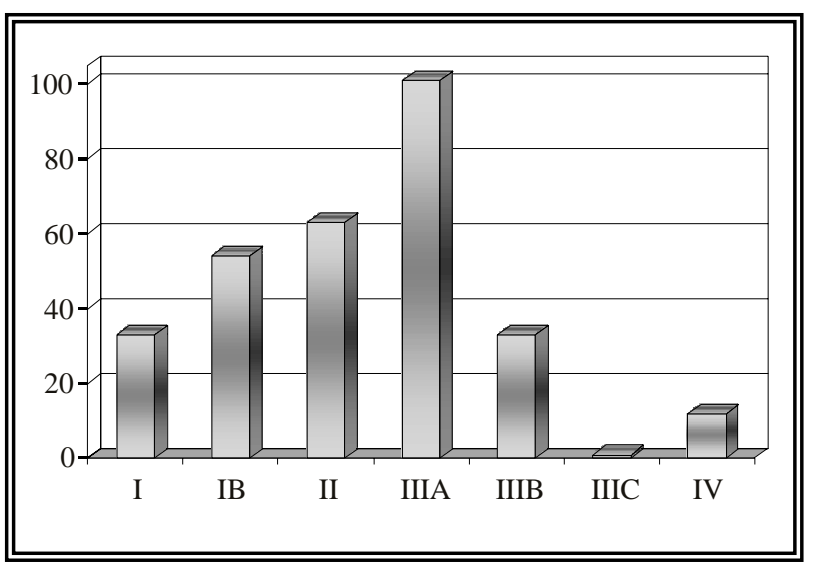

FIGURA 1. Distribución tumores en función del estadio (\%).

Para este estudio incluimos pacientes con tumores $\mathrm{pT}_{3 \mathrm{a}}$ por infiltración grasa (sin afectación adrenal), independientemente de la afectación ganglionar $\left(\mathrm{N}_{\mathrm{x}}\right)$ y con ausencia de metástasis. Los pacientes con tumores que infiltran la glándula adrenal y/o metástasis fueron descartados, al tener estos tumores un comportamiento biológico más agresivo que aquellos con afectación grasa exclusivamente ${ }^{5-7}$. Siguiendo estos criterios, de los 96 pacientes con afectación grasa fueron excluidos 4 por enfermedad a distancia, constituyendo una muestra homogénea de 92 pacientes. Las características clínico-tumorales de estos pacientes se recogen en la Tabla 1.

El estudio histopatológico de los tumores fue actualizado de acuerdo a la clasificación de 1997

Tabla 1

Características clínico-tumorales de los tumores $\mathrm{pT}_{3 \mathrm{a}} \mathrm{N}_{\mathrm{x}} \mathrm{M}_{0}$.

Características clínico-tumorales

\begin{tabular}{ll}
\hline Edad (mediana-rango) & $68(26-78)$ años \\
Varón & $59(64,13 \%)$ \\
Mujer & $33(35,86 \%)$ \\
Tamaño (mediana-rango) & $8(3-23) \mathrm{cm}$ \\
CR convencional & $66(71,8 \%)$ \\
CR papilar & $19(20,27 \%)$ \\
CR cromófobo & $2(2,2 \%)$ \\
CR indiferenciado & $5(5,4 \%)$ \\
Grado I-II & $55(59,78 \%)$ \\
Grado III-IV & $37(40,22 \%)$ \\
Progresión & $50(54,34 \%)$ \\
Tumores diploides & $34(50 \%)$ \\
Tumores aneuploides & $34(50 \%)$ \\
Afectación ganglionar & $23(25 \%)$ \\
\hline
\end{tabular}


(UIIC/AJCC) ${ }^{8}$. El grado nuclear se asignó según la clasificación de Fuhrman ${ }^{9}$. El tamaño tumoral fue considerado como el diámetro mayor del tumor en la pieza quirúrgica. Dentro de las características tumorales también fue estudiada la ploidía del ADN tumoral mediante citometría de flujo, siendo los histogramas valorables en 68 $(73,91 \%)$ de los 92 pacientes seleccionados (coeficiente de variación inferior a 8). Las muestras tumorales fueron consideradas diploides cuando el índice de ADN estaba entre 0,9 y 1,1 (cociente entre el canal del pico $G_{0} / G_{1}$ de la muestra y el canal del pico $G_{0} / G_{1}$ de las células control diploides normales). Además las muestras fueron consideradas diploides cuando sólo un pico era detectado en el análisis y aneuploides cuando se hallaban 2 picos.

El estado del paciente fue evaluado por última vez en marzo del 2005. El esquema de seguimiento incluyó análisis de sangre, radiografía de tórax, ecografía hepatorrenal y TAC abdominopélvico o RNM cada 6 meses durante los 2 primeros años. Posteriormente los controles radiológicos fueron anuales. La mediana de seguimiento fue establecida desde la fecha de intervención del paciente hasta la fecha última de revisión en consultas externas, siendo su valor de 72,5 meses (2-204). La progresión tumoral se definió como la recidiva locorregional y/o extensión a distancia. Actualmente permanecen vivos $47(51,1 \%), 37$ $(40,2 \%)$ han fallecido por causa tumoral, 1 está perdido para el seguimiento $(1,1 \%)$ y $7(7,6 \%)$ han fallecido por causa no tumoral.

Análisis estadístico: El análisis estadístico se realizó con programa estadístico SPSS versión 12.0 (SPSS, Inc., Chicago, Illinois). El valor de $\mathrm{p}$ considerado significativo fue menor de 0,05. El análisis de la supervivencia actuarial cáncer específica fue calculada con el método de KaplanMeyer y el test de log-rank para la comparación de grupos. Se obtuvieron curvas ROC en función del tamaño tumoral y el estado del paciente (vivo/muerto) en el momento de la revisión (Fig. 2). La curva seleccionó el mejor valor de corte para el tamaño tumoral (mejor sensibilidad y especificidad), que diferenció dos grupos con diferente pronóstico. Dicho punto de corte correspondió al valor $5,5 \mathrm{~cm}$, permitiendo dicotomizar y

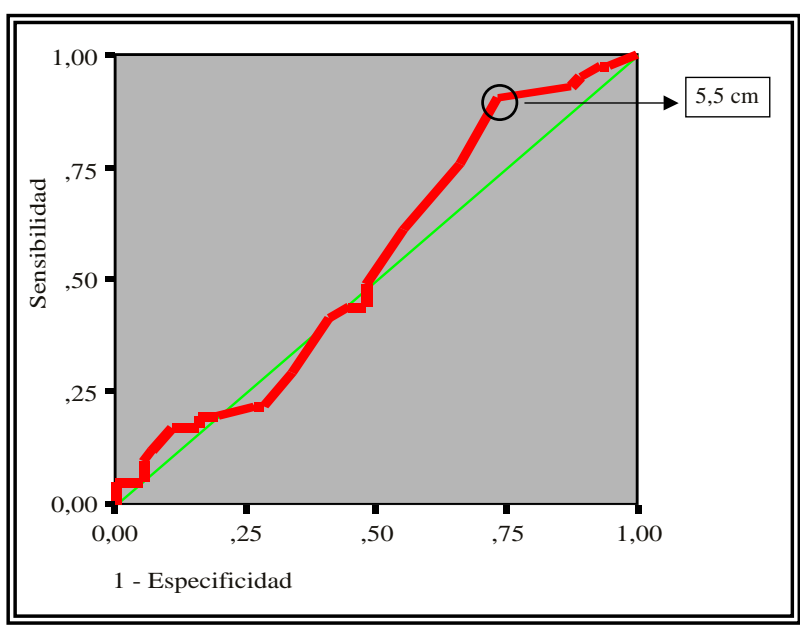

FIGURA 2. Curva ROC de sensibilidad especificidad.

definir dos grupos con diferencias significativas respecto a la supervivencia actuarial. Tras diferenciar estos dos grupos, se comparó el grupo menor o igual de $5,5 \mathrm{~cm}$, con el resto de subgrupos de la clasificación TNM, utilizando el método de Kaplan Meyer con el test de log-rank. Los puntos de corte 4 y $7 \mathrm{~cm}$ definidos en la clasificación TNM para los estadios $\mathrm{T}_{1} / \mathrm{T}_{2}$ fueron desestimados por la pérdida de sensibilidad y especificidad con respecto al valor $5,5 \mathrm{~cm}$ en nuestra muestra.

El análisis multivariante fue realizado mediante un modelo matemático de regresión logística, como método más adecuado para determinar la asociación entre un conjunto de variables explicativas continuas o categóricas y una variable respuesta de carácter binario (progresión tumoral). El análisis se realizó inicialmente para confirmar el valor pronóstico independiente del TNM en la progresión tumoral. Posteriormente se recalculó tras la reclasificación de los tumores $\mathrm{pT}_{3 \mathrm{a}} \mathrm{N}_{\mathrm{x}} \mathrm{M}_{0}$ menores o iguales de 5,5 $\mathrm{cm}$ como $\mathrm{pT}_{1}$, evaluando si el TNM mantenía su valor pronóstico independiente de progresión. Para dicho análisis, las variables fueron categorizadas siguiendo criterios clínicos y muestrales. Las variables incluidas en dicho análisis fueron el TNM, ploidía del ADN, tamaño tumoral, grado nuclear y edad.

\section{RESULTADOS}

La probabilidad de supervivencia de los pacientes en estadio pT3aNxMO a 3, 5 y 10 años fue de $59,96 \%, 53 \%$ y $47,97 \%$ respectivamente. 
La comparación de la supervivencia entre los distintos estadios TNM para toda la muestra (300 pacientes) mostró diferencias estadísticamente significativas entre las distintas categorías del TNM (test de log rank $\mathrm{p}<0,001$ ), tal y como se detalla en la Figura 3. Los tumores $\mathrm{pT}_{3 \mathrm{a}} \mathrm{Nx} \mathrm{MO}$, respecto a los tumores en estadio inferior $\left(\mathrm{pT}_{1-2}\right.$ $\mathrm{N}_{\mathrm{x}} \mathrm{M}_{0}$ ) mostraron peor supervivencia actuarial con diferencias estadísticamente significativas con el test de log-rank $(\mathrm{p}<0,001)$, como se puede apreciar en la Figura 4.

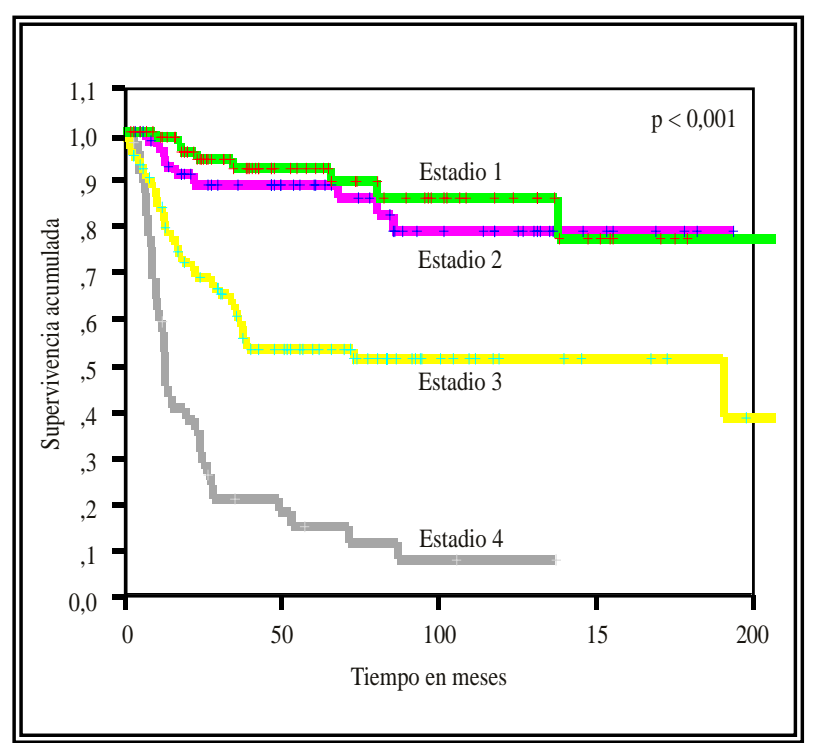

FIGURA 3. Comparación supervivencia actuarial en función del estadio tumoral.

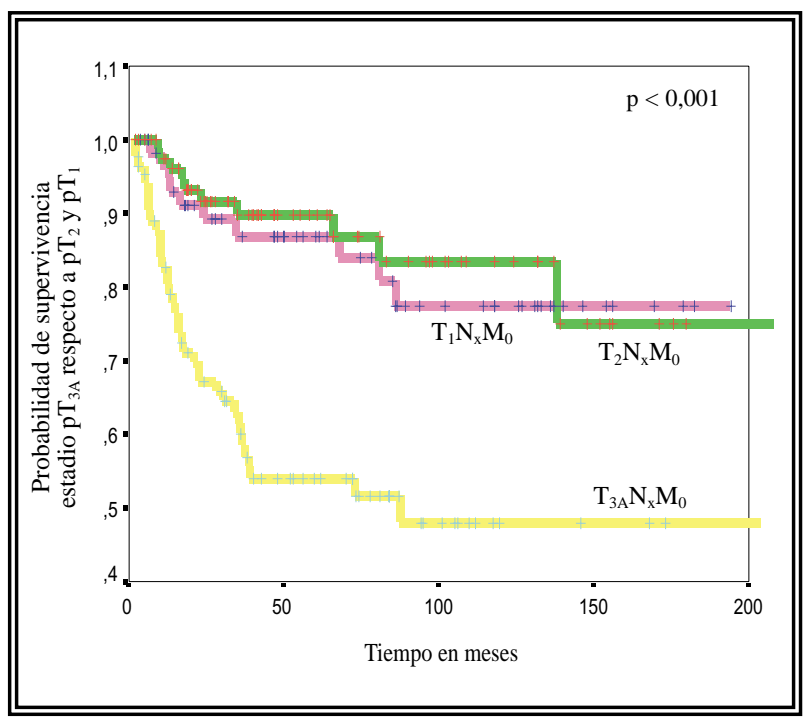

FIGURA 4. Comparación supervivencia actuarial del estadio $p T_{3 A}$ respecto a $p T_{2}$ y $p T 1$.
Los pacientes a estudio $\mathrm{pT}_{3 \mathrm{a}} \mathrm{N}_{\mathrm{x}} \mathrm{M}_{0}$ fueron dicotomizados en función del tamaño, según el mejor punto de corte obtenido con la curva ROC $(5,5 \mathrm{~cm})$. De los pT3a seleccionados, 18 pacientes $(19,52 \%)$ tenían un tamaño tumoral $\leq 5,5 \mathrm{~cm}$ y el resto, 74 $(80,43 \%)$ superior a $5,5 \mathrm{~cm}$. Las características tumorales de ambos grupos se detallan en la Tabla 2.

Tabla 2

Comparación de las características clínico-tumorales de los tumores $\mathrm{pT}_{3 \mathrm{a}} \mathrm{N}_{\text {cualquier }} \mathrm{M}_{0}<5,5 \mathrm{~cm}$ vs. $>5,5 \mathrm{~cm}$

\begin{tabular}{lcc}
\hline $\begin{array}{l}\text { Características clínico- } \\
\text { tumorales }\end{array}$ & $\begin{array}{c}\mathbf{P T}_{\mathbf{3 A}} \mathbf{N}_{\mathbf{X}} \mathbf{M}_{\mathbf{0}}< \\
\mathbf{5 , 5} \mathbf{~ c m}\end{array}$ & $\begin{array}{c}\mathbf{P T}_{\mathbf{3 A}} \mathbf{N}_{\mathbf{X}} \mathbf{M}_{\mathbf{0}}> \\
\mathbf{5 , 5} \mathbf{~ c m}\end{array}$ \\
\hline Pacientes n (\%) & $18(19,52)$ & $74(80,43)$ \\
Edad (mediana-rango) & $58(26-74)$ años & $62,5(30-81)$ años \\
CR convencional & $17(95,4 \%)$ & $49(66,21 \%)$ \\
CR papilar & $1(5,6 \%)$ & $18(24,32 \%)$ \\
CR cromófobo & $0(0 \%)$ & $2(3 \%)$ \\
CR indiferenciado & $0(0 \%)$ & $5(7,5 \%)$ \\
Grado I-II & $14(77,77 \%)$ & $41(55,40 \%)$ \\
Grado III-IV & $4(22,22 \%)$ & $33(44,59 \%)$ \\
Progresión & $5(27,8 \%)$ & $45(60,81 \%)$ \\
Tumores diploides & $6(46,2 \%)$ & $28(50,9 \%)$ \\
Tumores aneuploides & $7(53,8 \%)$ & $27(49,1 \%)$ \\
Afectación ganglionar & $0(0 \%)$ & $23(31,9 \%)$ \\
\hline
\end{tabular}

Al comparar la supervivencia actuarial entre ambos grupos (mayores vs. menores o iguales a $5,5 \mathrm{~cm}$ ), se observó mayor supervivencia en el grupo de menor tamaño tumoral con diferencias significativas (Fig. 5a). La supervivencia actuarial a 3 y 5 años fue de $82,64 \%$ y $75 \%$, en los pacientes con tumores $\leq 5,5 \mathrm{~cm}$, siendo $53,1 \%$ y $47,4 \%$ en los $>5 \mathrm{~cm}$ respectivamente. Se comparó entonces la supervivencia actuarial de los pT3a seleccionados con tamaño $\leq 5,5 \mathrm{~cm}$ con los tumores $\mathrm{pT}_{1-2} \mathrm{~N}_{\mathrm{x}} \mathrm{M}_{0}$ (Fig. 5b) siendo la supervivencia acumulada a 3 y 5 años de $89,77 \%$ y $86,87 \%$ para los

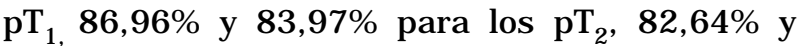
$75 \%$ para los pT3a $\leq 5,5 \mathrm{~cm}$, respectivamente. Estas diferencias no fueron estadísticamente significativas $(p=0,57)$. Cuando se compararon por separado, $\mathrm{pT}_{3 \mathrm{a}} \mathrm{N}_{\mathrm{x}} \mathrm{M}_{0} \leq 5,5 \mathrm{~cm}$ vs $\mathrm{pT}_{2} \mathrm{~N}_{\mathrm{x}} \mathrm{M}_{0}$ (Fig. 6) y $\mathrm{pT}_{3 a} \mathrm{~N}_{\mathrm{x}} \mathrm{M}_{0}$ vs $\mathrm{pT}_{1} \mathrm{~N}_{\mathrm{x}} \mathrm{M}_{0}$ (Fig. 7), tampoco se encontraron diferencias estadísticamente significativas en la supervivencia. 


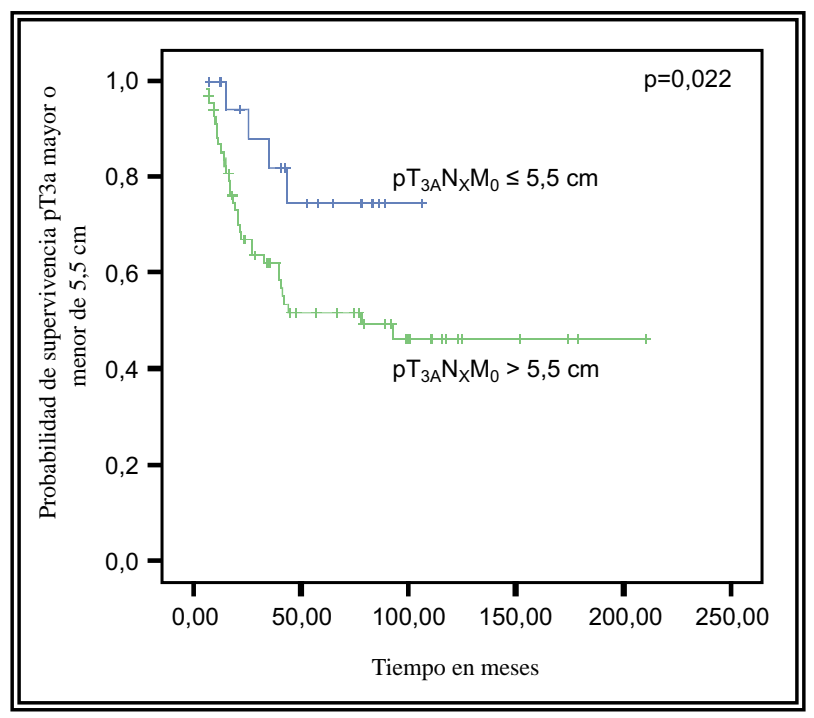

FIGURA 5a. Comparación supervivencia actuarial de los tumores $p T_{3 A} N_{X} M_{0} \leq 5,5 \mathrm{~cm}$ vs. $p T_{3 A} N_{X} M_{o}>5,5 \mathrm{~cm}$.

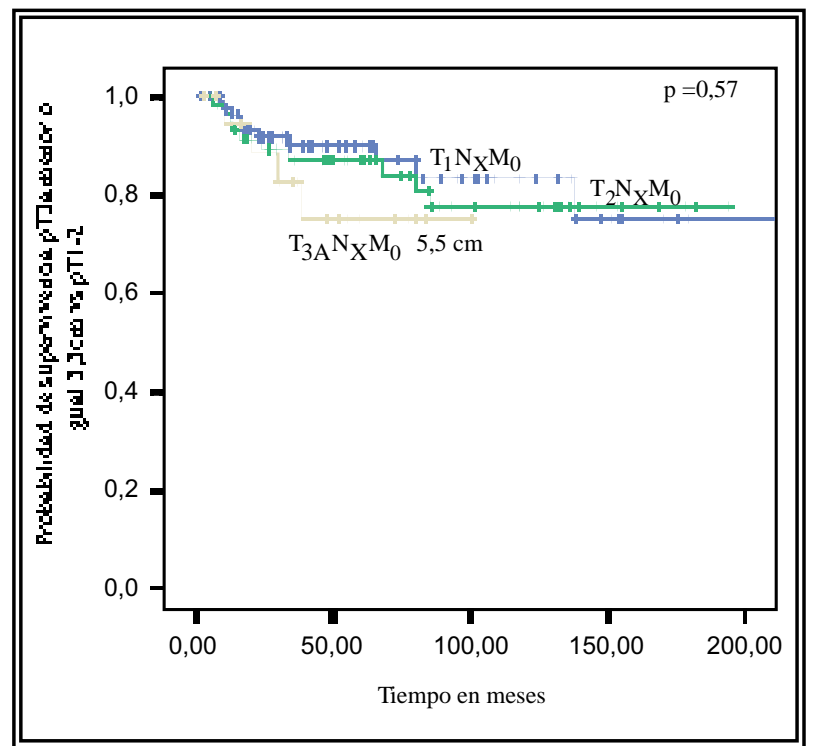

FIGURA 5b. Comparación supervivencia actuarial de los tumores $p T_{3 A} N_{X} M_{o} \leq 5,5 \mathrm{~cm}$ vs. $p T_{1-2} N_{X} M_{o}$.

Al no mostrar diferencias en cuanto a la supervivencia los $\mathrm{pT}_{3 \mathrm{a}} \leq 5,5 \mathrm{~cm}$ respecto a los $\mathrm{pT}_{1}$ (menores de $7 \mathrm{~cm}$ ), cabría preguntarse qué pasaría con el valor pronóstico de la clasificación TNM si reclasificábamos este subgrupo de tumores pT3aNxMo como pT1.

Para ello se realizó un análisis multivariante mediante regresión logística para evaluar los factores pronósticos de progresión tumoral (Tabla 3), previo y posterior a la reclasificación de los tumores $\mathrm{pT}_{3 \mathrm{a}} \mathrm{N}_{\mathrm{x}} \mathrm{M}_{0} \leq 5,5 \mathrm{~cm}$ como $\mathrm{pT}_{1}$. El primer análi-

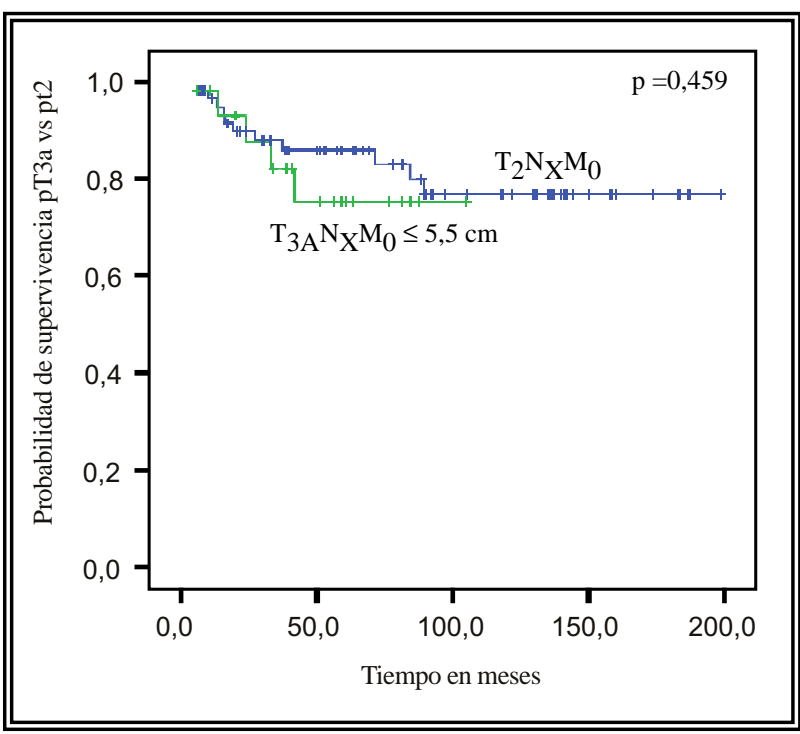

FIGURA 6. Comparación supervivencia actuarial de los tumores $p T_{3 A} N_{x} M_{0} \leq 5,5 \mathrm{~cm}$ vs. $p T_{2} N_{x} M_{0}$.

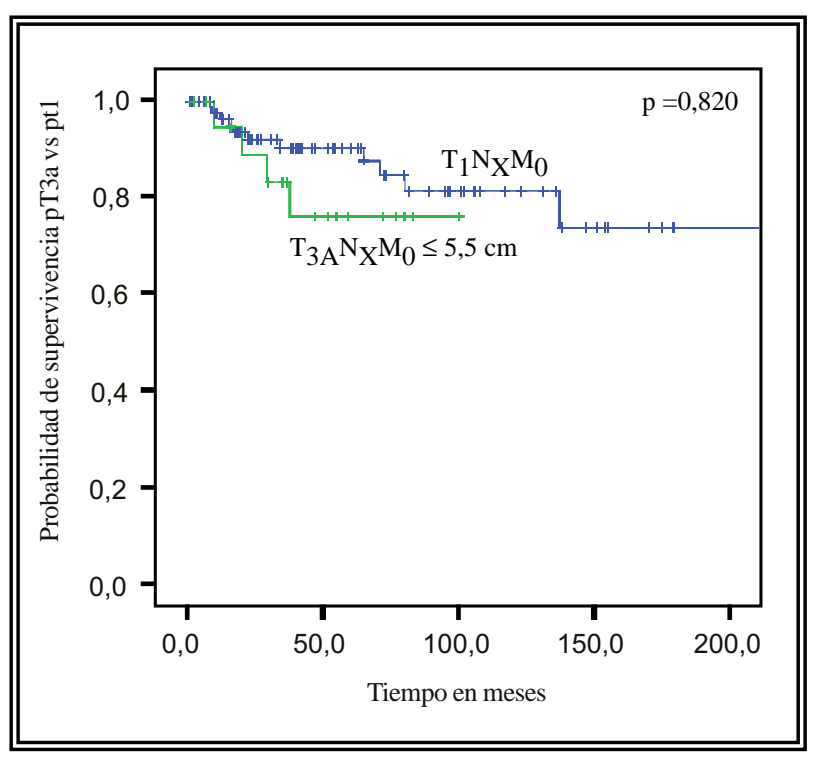

FIGURA 7. Comparación supervivencia actuarial de los tumores $p T_{3 A} N_{X} M_{O} \leq 5,5 \mathrm{~cm}$ vs. $p T_{1} N_{X} M_{O}$.

Tabla 3

Resultados del análisis de regresión logística, en relación a la progresión tumoral.

\begin{tabular}{lccccc}
\hline \multicolumn{6}{c}{ Progresión tumoral } \\
\hline Variables & Coef. & EE* & GL $^{\#}$ & P $^{\wedge}$ & OR (IC 95\%) \\
\hline TNM & 1,14 & 0,33 & 1 &, 0006 & $3,14(1,64-6,02)$ \\
Ploidia del ADN & 0,72 & 0,30 & 1 &, 01 & $2,07(1,14-3,76)$ \\
Grado nuclear & 0,72 & 0,31 & 1 &, 021 & $2,06(1,11-3,84)$ \\
\hline
\end{tabular}

M. predictivo $=67,51 \%$.

*Error estandar. ${ }^{*}$ Grado libertad. ^Significación. \Odd ratio. 
sis se realizó utilizando como variables explicativas, la clasificación TNM, la ploidía del ADN, el grado nuclear, tamaño tumoral y edad. Los resultados se muestran en la Tabla 3. De acuerdo con los coeficientes obtenidos, el riesgo de progresión varía dependiendo de la variable seleccionada. Así, los pacientes con tumores no órganoconfinados, tenían un riesgo de progresión tres veces mayor que aquellos con tumores organoconfinados. Los pacientes con tumores aneuploides tenían un riesgo dos veces mayor de progresión que aquellos con tumores diploides. De la misma forma, los pacientes con tumores con grado III-IV tenían un riesgo de progresión dos veces mayor que aquellos con grado I-II. Por tanto, de estas tres variables, el TNM es el que presentó mayor valor predictivo.

A partir de los resultados del primer análisis de regresión logística, calculamos la probabilidad estimada de progresión para cada grupo, comparando la realidad observada y la predicha por el modelo. La Tabla 4 refleja los casos clasificados correcta e incorrectamente. Por tanto, usando la información predictiva de las tres variables, el porcentaje de casos asignados correctamente fue del $67,51 \%$. La mejor predicción correspondió al grupo de tumores que no progresaron $(83,56 \%)$.

El segundo análisis se realizó reclasificando los tumores $\mathrm{pT}_{3 \mathrm{a}} \mathrm{N}_{\mathrm{x}} \mathrm{M}_{0}$ menores o iguales de 5,5 $\mathrm{cm}$ como $\mathrm{pT}_{1}$. El TNM conservó su valor predictivo, al igual que el grado y la ploidía del ADN, tal y como se muestra en la Tabla 5. La probabilidad estimada de progresión para cada grupo, comparando la realidad observada y la predicha por el modelo, tampoco se modificó.

\section{DISCUSIÓN}

La infiltración del tejido adiposo perinéfrico ha demostrado una disminución del 15 al 20\% de la supervivencia a 5 años, reflejando un fenotipo tumoral más agresivo. La afectación de la grasa próxima al seno renal parece presentar una mayor predilección por la infiltración venosa o por las metástasis a distancia al tener mejor acceso al sistema vascular ${ }^{10,11}$. Así, algunos estudios han determinado que la infiltración grasa es una variable pronóstica independiente de supervivencia ${ }^{12,13}$. Sin embargo, nosotros en base a nuestros resultados, pensamos que este dato tiene relevancia a la hora del análisis histopatológico, pero escasa repercusión en la práctica clínica.

Por tanto, cabe preguntarse qué significado tiene la infiltración grasa en el cáncer renal. La infiltración tumoral en la grasa renal (estadio $\mathrm{pT}_{3 \mathrm{a}}$ ) suele ser diagnosticada tras el análisis histopatológico de la pieza, sin repercusión en el planteamiento quirúrgico inicial ${ }^{14}$. Siemer ${ }^{7}$, en su serie de 12 pacientes con cáncer renal en estadio $\mathrm{pT}_{3 \mathrm{a}}$ en los que realizaron nefrectomía parcial basándose en el tamaño tumoral, la infiltración grasa no fue evidenciada por pruebas de imagen ni durante la cirugía. La supervivencia actuarial a 5 años fue comparable a la de los pacientes en estadio $\mathrm{pT}_{1} / \mathrm{pT}_{2}{ }^{15}$. En una serie más amplia de 485 nefrectomías parciales electivas según tamaño tumoral, los 81 pacientes con CR en estadio $\mathrm{pT}_{3 \mathrm{a}}$ por infiltración grasa tuvieron una supervivencia actuarial a 10 años comparable a la de los pacientes con tumores en estadio $\mathrm{pT}_{1} / \mathrm{pT}_{2}$. Por tanto, el tamaño tumoral, más que el hecho de infiltrar grasa perirrenal, debería ser el indicador del tipo de cirugia ${ }^{16}$. Nuestros datos también sugieren que el tamaño tumoral debería ser el factor esencial desde el punto de vista clínico y de planificación de tratamiento en tumores en estadio $\mathrm{pT}_{3 \mathrm{a}}$ por infiltración grasa y ausencia de metástasis.

El tamaño tumoral se ha relacionado con la aparición de metástasis y con la supervivencia a

Tabla 4

Comparación entre los grupos observados y previstos por el modelo de regresión logística.

\begin{tabular}{lccc}
\hline \multicolumn{4}{c}{ Predicho por el modelo } \\
\hline Observados & Progresión & No progresión & \% Correcto \\
\hline Progresión & 38 & 53 & $41,75 \%$ \\
No progresión & 24 & 122 & $83,56 \%$ \\
\hline
\end{tabular}

\section{Tabla 5}

Resultados del análisis de regresión logística, en relación a la progresión tumoral, tras reclasificar los tumores pT3a Nx M0 menores o iguales de 5,5 como pT1.

\begin{tabular}{lccccc}
\hline Progresión tumoral & & & & \\
\hline Variables & Coef. & EE & GL & P & RR (IC 95\%) \\
\hline TNM & 1,146 & 0,331 & 1 &, 0006 & $3,14(1,64-6,02)$ \\
Ploidia del ADN & 0,729 & 0,303 & 1 &, 0161 & $2,07(1,14-3,76)$ \\
Grado nuclear & 0,729 & 0,316 & 1 &, 0215 & $2,06(1,11-3,84)$ \\
\hline
\end{tabular}


largo plazo. Así Bell ${ }^{17}$, ya evidenció una incidencia de metástasis en tumores mayores de $10 \mathrm{~cm}$ del $85 \%$, y del $11 \%$ en menores de $3 \mathrm{~cm}$. Giliani et al. ${ }^{18}$, observaron tasas de supervivencia a 5 años del 0, 50 y 83,5\% en tumores mayores de 10 $\mathrm{cm}$, entre $5-10 \mathrm{~cm}$ y menores de $5 \mathrm{~cm}$ respectivamente. Este hecho se explica por la relación entre el tamaño y el estadio patológico, si bien algunos estudios han proporcionado al tamaño tumoral un valor pronóstico independiente para estadios avanzados ${ }^{1}$. Sin embargo, en tumores órganoconfinados el tamaño tumoral también muestra su valor pronóstico, con supervivencias superiores al $90 \%$ en tumores menores de $4 \mathrm{~cm}$ con independencia si son tratados con cirugía radical o parcial $^{19}$. Por todo ello, parece que el tamaño tumoral para cada uno de los estadios puede ser un factor pronóstico independiente ${ }^{20}$. En un estudio reciente, Frank et al. ${ }^{21}$, también determinaron la asociación del incremento del tamaño tumoral con la mayor probabilidad de tumores de alto grado nuclear.

Del mismo modo, el tamaño ha sido la variable fundamental para la clasificación tumoral en este trabajo. Tras la categorización de la muestra en función del tamaño, al reclasificar los tumores $\mathrm{pT}_{3 \mathrm{a}}$ con ausencia de metástasis de tamaño $\leq 5,5 \mathrm{~cm}$ como $\mathrm{pT}_{1}$, no se modificaba el valor pronóstico independiente de progresión de las variables TNM, grado nuclear y ploidía del ADN en el análisis multivariante. Estos resultados están en la línea de los publicados por Siemer et al. ${ }^{7}$, quienes obtuvieron similares conclusiones al categorizar su muestra de 237 tumores $\mathrm{pT}_{3 \mathrm{a}}$ por infiltración grasa en función del tamaño tumoral, con su punto de corte óptimo fijado en $7 \mathrm{~cm}$ obtenido mediante curvas ROC. Esta diferencia en el punto de corte puede deberse a las diferencias en el tamaño de la muestra. Serían necesarios más estudios con mayores tamaños muestrales para fijar el punto de corte óptimo del tamaño y una validación externa antes de modificar esta categoría del TNM.

A la vista de nuestros resultados, una clasificación TNM modificada que reclasificara a los tumores con infiltración grasa en función del tamaño, clasificando a los tumores $\leq 5,5 \mathrm{~cm}$. como $\mathrm{pT}_{1}$ y a los mayores de $5,5 \mathrm{~cm}$ como $\mathrm{pT}_{3 \mathrm{a}}$, no afectaría al valor pronóstico independiente de progresión de la clasificación TNM. Esta reclasificación permitiría una mejor predicción pronóstica del paciente, facilitando la decisión terapeútica en función del tamaño.

\section{CONCLUSIONES}

Los tumores de células renales $\mathrm{pT}_{3 \mathrm{a}} \mathrm{N}_{\mathrm{x}}$ por infiltración grasa sin metástasis de tamaño $\leq 5,5$ $\mathrm{cm}$, se comportan como tumores organoconfinados respecto a la supervivencia y su reclasificación no modifica el valor pronóstico independiente del TNM como predictor de progresión tumoral.

\section{REFERENCIAS}

1. Guinan P, Sobin LH, Algaba F, Badellino F, Kameyama S, MacLennan G, et al. TNM staging of renal cell carcinoma: Workgroup No. 3. Union International Contre le Cancer (UICC) and the American Joint Committee on Cancer (AJCC). Cancer 1997;80:992-993.

2. Javidan J, Stricker HJ, Tamboli P, Amin MB, Peabody JO, Deshpande A, et al. Prognostic significance of the 1997 TNM classification of renal cell carcinoma. J Urol 1999;162: 1277-1281.

3. Greene FL, Sobin LH. The TNM system: our language for cancer care. J Surg Oncol 2002;80:119-120.

4. Han KR, Bui MH, Pantuck AJ, Freitas DG, Leibovich BC, Dorey FJ, et al. TNM T3a renal cell carcinoma: adrenal gland involvement is not the same as renal fat invasion. $J$ Urol 2003;169:899-903; discussion 903-894.

5. Mejean A, Hopirtean V, Bazin JP, Larousserie F, Benoit H, Chretien Y, et al. Prognostic factors for the survival of patients with papillary renal cell carcinoma: meaning of histological typing and multifocality. J Urol 2003;170:764-767.

6. Sánchez Zalabardo D, Arocena García-Tapia J, Regojo Balboa JM, Fernández Montero JM, López Ferrandis J, Rosell Costa D, et al. Prognosis factors in pT3 renal carcinoma. Actas Urol Esp 2003;27:26-32.

7. Siemer S, Lehmann J, Loch A, Becker F, Stein U, Schneider G, et al. Current TNM classification of renal cell carcinoma evaluated: revising stage T3a. J Urol 2005;173:33-37.

8. Storkel S, Eble J, Adlakha K, Amin MB, Blute M, Bostwick D. Classification of renal cell carcinoma: workgroup $\mathrm{n}^{\mathrm{o}} 1$. Union Internationale Contre Le Cancer (UICC) and the American Joint Committeen on Cancer (AJCC). Cancer 1997;80:987-992.

9. Fuhrman S, Lasky L, Limas C. Prognostic significance of morphologic parameters in renal cell carcinoma. Am J Surg Pathol 1982;6:655-661.

10. Thompson R, Leibovich B, Cheville J, WS. W, Louse C, Kwon $\mathrm{E}$, et al. Is renal sinus fat invasion the same as perinephric fat invasion for pT3a renal cell carcinoma? J Urol 2005; 174:1218-1221.

11. Bonship S, Gibson D, Mhoon M, Greene G. Renal sinus involvement in renal cell carcinomas. Am J Surg Pathol 2000;24:451-458.

12. Ficarra V, Prayer-Galetti T, Novella G, Bratti E, Maffei N, Dal Bianco $M$, et al. Incidental detection beyond pathological factors as prognostic predictor of renal cell carcinoma. Eur Urol 2003;43:663-669. 
13. Patard JJ, Leray E, Rodríguez A, Rioux-Leclercq N, Guille F, Lobel B. Correlation between symptom graduation, tumor characteristics and survival in renal cell carcinoma. Eur Urol 2003;44:226-232.

14. Hofmockel G, Tsatalpas P, Muller H, Dammrich J, Poot M, Maurer-Schultze B, et al. Significance of conventional and new prognostic factors for locally confined renal cell carcinoma. Cancer 1995;76:296-306.

15. Hafez KS, Fergany AF, Novick AC. Nephron sparing surgery for localized renal cell carcinoma: impact of tumor size on patient survival, tumor recurrence and TNM staging. J Urol 1999;162:1930-1933.

16. Fergany AF, Hafez KS, Novick AC. Long-term results of nephron sparing surgery for localized renal cell carcinoma: 10-year followup. J Urol 2000;163:442-445.

17. Bell E. A classification of renal tumours with observations on the frecuency of the various types. J Urol 1938;39:238241.
18. Giuliani L, Giberti C, Martorana S, Rovida S. Radical extensive surgery for renal cell carcinom: long term results and prognostic factors. J Urol 1990;143:468-473.

19. Butler B, Novick A, Muller D. Management of small unilateral renal cell carcinoma: radical versus nephron sparing surgery. Urology 1995;45:34-40.

20. Kontak J, Campbell S. Prognostic factors in renal cell carcinnoma. Urol Clin North Am 2003;30:467-480.

21. Frank I, Blute ML, Cheville JC, Lohse CM, Weaver AL, Leibovich BC, et al. A multifactorial postoperative surveillance model for patients with surgically treated clear cell renal cell carcinoma. J Urol 2003;170:2225-2232.

Dr. L. Gómez Pérez

E-mail: luisgope@hotmail.com

(Trabajo recibido el 13 de octubre 2005) 